news and views

\title{
Differential attack
}

\author{
from Robert W. Cahn
}

THE essentially empirical science of chemical etching, used as a routine tool by the material scientist to reveal the microstructure of mineral samples, has developed in some unexpectedly sophisticated ways. One is the recent development of a thin-film ultramicrosieve (Kitada J. mater. Sci. 14, 2765; 1979), the latest in a line of filters whose unlikely origins lie in an observation made years ago that chemical etching could reveal the damage tracks left by the passage of nuclear fission fragments through thin mica sheets.

This story begins in 1961, when R.M. Walker and P.B. Price at the Central General Electric Research Laboratory in Schenectady discovered that these damage tracks were revealed by chemical etching which pierced minute, uniformly-sized holes through the mica. Other minerals were soon found to behave similarly, and other forms of radiation found sometimes to yield etchable tracks. Walker and Price, aided by R.L. Fleischer, over the next few years applied this differential etching method to many problems in geochronology, planetary physics (the study of meteorites in particular), palaeontology and nuclear physics. The field has become large enough to achieve that ultimate accolade, a journal of its own.

The early mica work came to the attention of S.H. Seal, a clinical cancer researcher at the Sloan-Kettering Institute who needed an ultrafilter to hold back cancer cells from suspension in blood; nothing he had tried would do the trick. Price's irradiated and etched mica foils were just right (the holes were all 3-4 $\mu \mathrm{m}$ across) but the foils were too fragile. At this point, Fleischer found that a polycarbonate polymer could be substituted for mica and had better mechanical properties. Thus began the manufacture of GE's 'Nucleopore' biological filters, which, among other uses have a role in cancer diagnosis. The filters were described by Fleischer, Price and Symes in Science $(143,249 ; 1964)$, and the steps in this weird progression from fission damage research to clinical instrumentation are traced, step by step, in that excellent survey, Applied Science and
Technological Progress (313, NAS, Washington, 1967).

This case-history has evidently influenced subsequent research at GE. For instance, W. Desorbo and H.E. Cline, who were investigating directionally solidified eutectics, consisting of parallel metallic rods in a matrix, with a view to their use in jet engine turbine blades, found that differential etching left the rods protruding clear of the matrix. Porous metallic replicas made from such etched samples performed neatly as microsieves (J. appl. Phys. 41, 2099; 1970).

Filters with holes a few microns across are considered coarse nowadays (too coarse, for example, for the isolation of airborne smokes), and a new approach has now shown how to make filters with submicron apertures. M. Kitada ( $J$. mater. Sci. $o p$. cit.) co-evaporated gold and germanium in vacuum onto sodium chloride crystals. Films $50-500 \mathrm{~nm}$ thick were evaporated, at or near the eutectic composition of 12 at \% Ge. Germanium was deposited as round grains $20-250 \mathrm{~nm}$ across, depending on alloy composition and substrate temperature. The films were floated off in water and attacked by an iodated mixed-acid etchant, which selectively removed the more reactive germanium. Since the germanium grains grow in columnar fashion through the film thickness, removal of germanium leaves minute pores right through the residual gold films and the result is a thin-film ultramicrosieve.

Another form of selective attack which is not only technologically valuable but scientifically intriguing, is the vertical etching of silicon. This technique, which for several years has been quietly developed within the semiconductor industry, is now beginning to find applications in other scientific fields. An excellent account of the technique, its scientific basis and recent very various applications has just been published by D.L. Kendall ( $A$. Rev. Mater. Sci. 9,373 ; 1979) who regards it as a 'whole new way of structuring and using a solid material'. The essential fact is that extremely narrow, deep grooves with depth-to-width ratios exceeding 100:1 can be etched in silicon. It is only necessary to start with a monocrystal slice accurately parallel to (110), and to prepare an oxide surface mask with narrow gaps precisely parallel to the $(110) /(1 \overline{1} 1)$ intersection. Etching is very much faster in the [110] than in the [111] direction, because the newly formed (111) plane is quickly covered with an oxide film which severely hinders access of etchant (usually an aqueous solution of potassium hydroxide used at $80^{\circ} \mathrm{C}$ ), whereas other planes are not thus affected. Any divergence of the surface slit from correct alignment enhances the rate of etching of the sides of the groove.

Whereas it is easy to etch deep ultranarrow grooves, it is not possible to etch round holes. A regular array of exact $5 \mu \mathrm{m}$ square holes has been fabricated by etching two populations of grooves, mutually inclined at $70^{\circ}$, from opposite sides of a (110) slice until they just meet (Bean, IEEE Trans. ED-25, 1185; 1978). Other uses include deeply etched solar energy absorbers (effectively thin-layer blackbodies), an elaborate high-area $\mathrm{Si} / \mathrm{SiO}_{2}$ capacitor only about $1 \mathrm{~mm}$ square, diffraction gratings efficient for highorder optical spectra and an infrared reflection polariser. The most subtle application is a device for ejecting inkdrops between closely spaced pairs of electrified $p / n$ junctions to charge the drops, which can subsequently be electrically steered to form a printed image. This has cleared the way to computercontrolled printing without moving parts.

A variant on the sieve theme is a selective-ion device for separating ions such as $\mathrm{Cu}^{+}$, which diffuse very fast in silicon. A thin-walled meander is formed in a silicon slice by etching offset parallel grooves from the two sides; copper will pass selectively through such a silicon membrane, much as hydrogen passes selectively through palladium. If the membrane is oxidised by anodising in hydrofluoric acid, the oxide has pores which can be as small as $1 \mathrm{~nm}$ in diameter. These can be used to separate molecules of different sizes: larger pores, for example, can serve as virus filters (Watanabe et al. $J$. Electrochem. Soc. 122, 1351; 1975; Gregor \& Gregor Sci. Am. 239, 88; 1978).

Robert W. Cahn is Professor of Materials Science in the University of Sussex. 